\title{
PARÁMETROS RELEVANTES DE LA CURVA ESFUERZO- DEFORMACIÓN EN COMPRESIÓN DE CONCRETOS NO CONFINADOS PRODUCIDOS EN MÉXICO
}

\author{
Ramón Rodelo(l), Mario E. Rodríguez ${ }^{(1)}$ y José I. Restrepo ${ }^{(2)}$
}

\begin{abstract}
RESUMEN
Las Normas Técnicas Complementarias para Diseño por Sismo para la Ciudad de México especifica por primera vez, métodos de análisis sísmico no lineal de estructuras, para los cuales es necesario conocer, entre otros, los parámetros que definen la curva-esfuerzo-deformación en compresión del concreto sin confinar. En esta investigación se llevaron a cabo estudios analíticos y experimentales de concretos producidos recientemente en México. Con base en los resultados encontrados se dan recomendaciones para estimar parámetros que definen de manera importante la curva esfuerzodeformación del concreto sin confinar. Los estudios experimentales de los especímenes de concreto se realizaron en el Instituto de Ingeniería de la UNAM, aplicando ciclos de carga en compresión de acuerdo con las normas ASTM y NMX correspondientes y tomando como resultado un promedio de las muestras ensayadas. Se proponen expresiones para obtener el módulo de elasticidad del concreto, $E_{c}$, parámetro que es relevante en el cómputo de desplazamientos laterales de estructuras en zonas sísmicas, así como en el cómputo de los periodos de vibrar de una estructura en estas zonas. También en esta investigación se dan expresiones para obtener valores de parámetros importantes de la curva esfuerzo-deformación del concreto no confinado en compresión, los cuales se emplean en el modelado de articulaciones plásticas para el análisis no lineal. Estos parámetros son la relación de $E_{c}$ y el módulo secante, $E_{s e c}, E_{c} / E_{s e c}$ y la deformación del concreto en $f^{\prime}{ }_{c}, \varepsilon^{\prime}$. Con base en los resultados de estos ensayes se dan recomendaciones para la estimación de estos parámetros para concretos producidos recientemente en México.
\end{abstract}

Palabras clave: Concreto; resistencia a compresión; curva esfuerzo-deformación; módulo de elasticidad; módulo secante; peso volumétrico

\section{RELEVANT PARAMETERS OF THE STRESS-DEFORMATION CURVE IN COMPRESSION OF UNCONFINED CONCRETS PRODUCED IN MEXICO}

\begin{abstract}
The seismic design standard for the city of Mexico prescribes the use of no-lineal analyses, for which it is necessary to define, among various others, the parameters of the unconfined concrete stress-strain

Artículo recibido el 5 de diciembre de 2018 y aprobado para su publicación el 24 de agosto de 2020. Se aceptarán comentarios y/o discusiones hasta cinco meses después de su publicación.

(1) Instituto de Ingeniería, Universidad Nacional Autónoma de México, Coyoacán, C.P. 04510, México, Ciudad de México. ramonrra@hotmail.com; mrod@unam.mx

(2) Universidad de California, San Diego. jrestrepo@ucsd.edu
\end{abstract}

DOI: $10.18867 /$ ris.103.534 
relationship. This study describes results of analytical and experimental studies of unconfined concrete produced in Mexico. Based on experimental test data obtained in this study, recommendations for estimating parameters that define the unconfined concrete stress-strain curve are given. Test of concrete cylinders were conducted at the Instituto de Ingeniería (Institute of Engineering), UNAM, México, following testing procedures according to national and international standards. and taking average results of tested samples. In this research, expressions for determining the modulus of elasticity $E_{c}$, are given. Parameter $E_{c}$ is relevant for computing lateral displacements of structures subjected to earthquake actions, as well as for computing periods of vibration of these structures. It is also presented expressions to define other important parameters of the concrete stressstrain curve, which are considered when modeling plastic hinges in nonlinear analysis. Such parameters are the strain at the stress $f_{c}^{\prime}$ of unconfined concrete specimen, $\varepsilon_{c}^{\prime}$, and the ratio of $E_{c}$ to the secant modulus $E_{\text {sec }}$. Recommendations are given for estimating these parameters for concrete produced in Mexico.

Keywords: Concrete; compressive stress; modulus of elasticity; secant modulus; compressive stressstrain curve; density of concrete measure

\section{INTRODUCCIÓN}

Las Normas Técnicas Complementarias para Diseño por Sismo para la Ciudad de México (NTCS, 2017) especifica por primera vez, métodos de análisis sísmico no lineal de estructuras, para los cuales es necesario conocer los parámetros que definen la curva-esfuerzo-deformación en compresión del concreto sin confinar, $f_{c}-\varepsilon_{c}$. Adicionalmente, las normas de diseño sísmico para la Ciudad de México, y de otros estados en el país, especifican parámetros como el módulo de elasticidad, $E_{c}$, para el análisis sísmico de estructuras. Este parámetro se emplea tanto para el cómputo de desplazamientos laterales de estructuras, como para conocer los periodos de vibrar de estructuras. El módulo de elasticidad es relevante en el cómputo de desplazamientos laterales, el sobrestimar el valor de este parámetro llevaría a subestimar los valores de desplazamientos laterales para las fuerzas sísmicas correspondientes al sismo de diseño, y por tanto a subestimar el posible daño en la estructura. Además, un valor incorrecto de $E_{c}$ llevaría también a obtener valores erróneos de periodos de vibrar de la estructura, y por tanto valores incorrectos de las fuerzas sísmicas de diseño en el análisis modal espectral que usualmente se lleva a cabo en los despachos de diseño estructural.

La fig. 1 muestra una curva típica esfuerzo-deformación del concreto expresada en forma adimensional con la relación $f_{c} / f^{\prime}{ }_{c}$ para los esfuerzos, y $\varepsilon_{c} / \varepsilon^{\prime}{ }_{c}$ para las deformaciones, donde $f_{c}$ y $\varepsilon_{c}$ son el esfuerzo y deformación del concreto en cualquier punto de la curva, $f_{c}^{\prime}$ es la resistencia en compresión especificada del concreto y $\varepsilon_{c}{ }_{c}$ es la deformación del concreto correspondiente a la resistencia en compresión $f_{c}^{\prime}$. Uno de los parámetros más importantes que permite definir la curva $f_{c}-\varepsilon_{c}$ es el módulo de elasticidad, $E_{c}$. Para el cálculo de $E_{c}$ se tienen expresiones en las Normas Técnicas Complementarias de la Ciudad de México (NTC, 2017); sin embargo, estas expresiones se obtuvieron de estudios de concretos producidos en los años 80's en la Ciudad de México. Para el resto de las regiones en México estas expresiones no serían aplicables. Además, los estudios que respaldan estas expresiones datan de los años 80 's, donde concretos a partir de $f_{c}^{\prime}=40 \mathrm{MPa}$ se consideraban de alta resistencia. En la actualidad, en edificaciones del país se utilizan en algunos casos concretos de hasta $f^{\prime}{ }_{c}=100 \mathrm{MPa}$. Esto indica que es necesario contar con expresiones para definir parámetros relevantes de la curva $f_{c}-\varepsilon_{c}$ para concretos que se producen actualmente en México. Estos parámetros, además de $E_{c}$, son la deformación unitaria asociada al esfuerzo máximo $\varepsilon^{\prime}$, deformación última del concreto $\varepsilon_{c u}$, así como la relación entre $E_{c}$ y el módulo secante, $E_{s e c}, E_{d} / E_{s e c}$, donde $E_{s e c}$ se define como $f^{\prime}{ }_{c} / \varepsilon_{c}^{\prime}$. La fig. 2 muestra de manera gráfica los parámetros $E_{c}$ y $E_{s e c}$. Expresiones que 
permitan el cómputo de parámetros como $\varepsilon_{c}^{\prime}$ y $E_{s e c}$ son especialmente relevantes en el análisis no lineal de estructuras de concreto reforzado, ya que éste es un método de análisis especificado por las actuales Normas Técnicas Complementarias para Diseño por Sismo (NTCS, 2017).

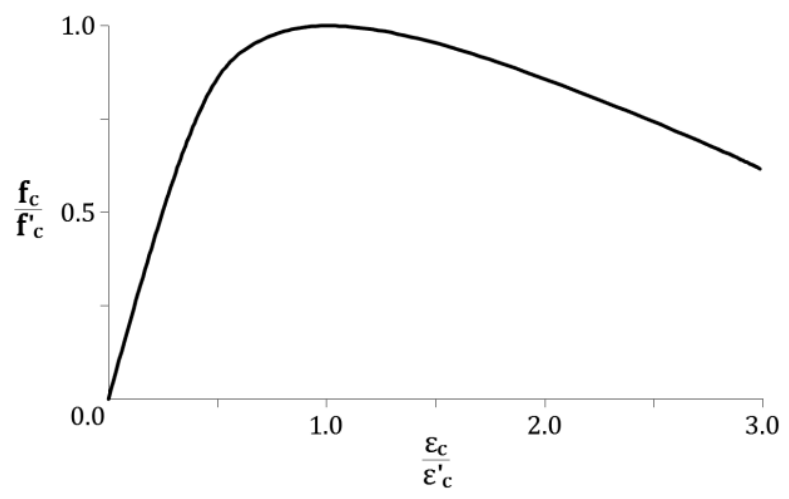

Figura 1. Curva adimensional esfuerzo-deformación del concreto sin confinar

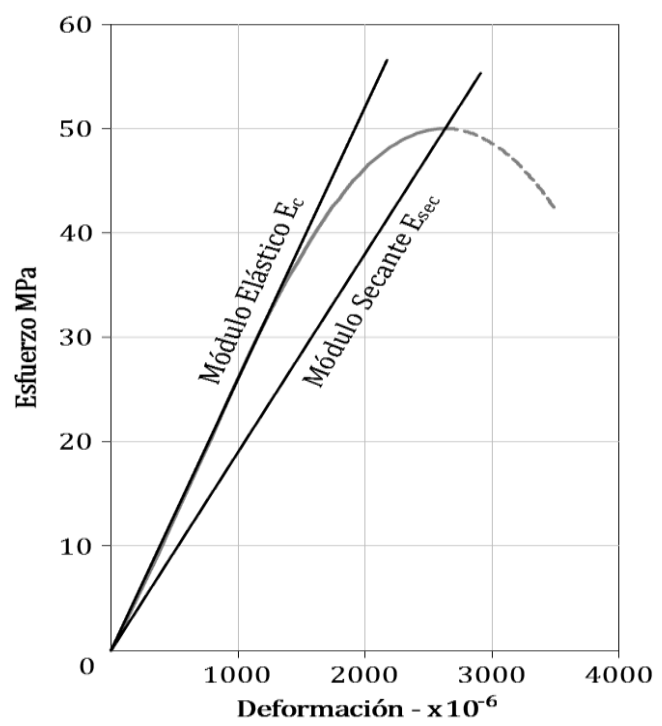

Figura 2. Curva esfuerzo-deformación del concreto y parámetros $E_{c}$ y $E_{s e c}$.

En la presente investigación se pretende proponer expresiones de parámetros importantes que definen la curva esfuerzo-deformación del concreto no confinado, que consideren las propiedades de los concretos en el país. Estos parámetros no se conocen en México, por lo que los diseños sísmicos de estructuras que emplean el módulo elástico $E_{c}$ están limitados, tanto para un análisis elástico lineal como también para análisis del tipo inelástico. También los estudios que se desarrollan en México con expresiones para la curva $f_{c}-\varepsilon_{c}$ del concreto en compresión para el proceso de diseño sísmico de estructuras requieren conocer valores apropiados de los parámetros que definen esta curva.

Las muestras para este estudio fueron donadas por la AMICP (Asociación Mexicana de la Industria del Cemento Premezclado A.C.) y se tomaron utilizando una zonificación de regiones considerando solo los agregados con los que se pueden fabricar concretos para uso estructural (Clase $I$ ) especificados por las Normas Técnicas Complementarias de la Ciudad de México (NTC, 2017). Los ensayes realizados en el laboratorio siguieron lo especificado en las normas ASTM-469 (2002) y NMX-C-128 (1997). 


\section{REVISIÓN DEL ESTADO DEL ARTE}

\section{Módulo de elasticidad $\boldsymbol{E}_{c}$}

Mendoza (1985), realizó una de las primeras investigaciones relacionadas con las propiedades mecánicas de los concretos de la Ciudad de México. Este autor propuso expresiones para $E_{c}$ en función de la raíz cuadrada de la resistencia del concreto, basándose en la combinación de los tipos de agregados de los concretos, obteniendo las siguientes expresiones para los concretos de la Ciudad de México.

Para concretos con grava y arena andesítica:

$$
\begin{aligned}
& E_{c}=2500 \sqrt{f_{c}^{\prime}}, \quad E_{c} \mathrm{y} f_{c}^{\prime} \text { en } \mathrm{MPa} \\
& \left(E_{c}=8000 \sqrt{f_{c}^{\prime}}, \quad E_{c} \mathrm{y} f_{c}^{\prime} \text { en } \mathrm{kg} / \mathrm{cm}^{2}\right)
\end{aligned}
$$

Para concretos con grava basáltica y arena andesítica:

$$
\begin{aligned}
& E_{c}=3500 \sqrt{f_{c}^{\prime}}, \quad E_{c} \text { y } f_{c}^{\prime} \text { en } \mathrm{MPa} \\
& \left(E_{c}=11000 \sqrt{f_{c}^{\prime}}, \quad E_{c} \text { y } f_{c}^{\prime} \text { en } \mathrm{kg} / \mathrm{cm}^{2}\right)
\end{aligned}
$$

y para grava ligera de arcilla expandida y arena andesítica:

$$
\begin{array}{ll}
E_{c}=2075 \sqrt{f_{c}^{\prime}}, & E_{c} \text { y } f_{c}^{\prime} \text { en } \mathrm{MPa} \\
\left(E_{c}=6500 \sqrt{f_{c}^{\prime}},\right. & \left.E_{c} \mathrm{y} f_{c}^{\prime} \text { en } \mathrm{kg} / \mathrm{cm}^{2}\right)
\end{array}
$$

El Comité ACI-363 del Instituto Americano del Concreto (ACI-363, 1992), propuso una aproximación para la estimación del módulo elástico de concretos en Estados Unidos, afectando la raíz cuadrada del $f^{\prime}{ }_{c}$ más un factor de ajuste por el peso volumétrico de los agregados de la muestra de concreto, obteniendo como resultado la ecuación:

$$
\begin{aligned}
& E_{c}=3320 \sqrt{f_{c}^{\prime}}+6900, \quad E_{c} \mathrm{y} f_{c}^{\prime} \text { en } \mathrm{MPa} \\
& \left(E_{c}=10600 \sqrt{f_{c}^{\prime}}+70400, \quad E_{c} \mathrm{y} f_{c}^{\prime} \text { en } \mathrm{kg} / \mathrm{cm}^{2}\right)
\end{aligned}
$$

El Comité ACI 318 del Instituto Americano del Concreto ACI-318-14 (ACI-318, 2014) establece desde hace años una expresión para la estimación del módulo de elasticidad del concreto a 28 días de edad, afectando la raíz cuadrada del $f^{\prime} c$ del concreto por un factor de ajuste donde se incluyen las propiedades del agregado a partir del peso volumétrico del concreto, $w_{c}$, como se observa en la siguiente expresión:

$$
\begin{array}{ll}
E_{c}=0.043 w_{c}^{1.5} \sqrt{f_{c}^{\prime}}, & E_{c} \text { y } f_{c}^{\prime} \text { en MPa y } w_{c} \text { en } \mathrm{kg} / \mathrm{m}^{3} \\
\left(E_{c}=0.14 w_{c}^{1.5} \sqrt{f_{c}^{\prime}},\right. & \left.E_{c} \mathrm{y} f_{c}^{\prime} \text { en } \mathrm{kg} / \mathrm{cm}^{2} \text { y } w_{c} \text { en } \mathrm{kg} / \mathrm{m}^{3}\right)
\end{array}
$$


Para concretos de peso normal, el ACI 318-14 especifica:

$E_{c}=4700 \sqrt{f_{c}^{\prime}}, \quad E_{c} \mathrm{y} f_{c}^{\prime}$ en MPa

$E_{c}=15000 \sqrt{f_{c}^{\prime}}, \quad E_{c} \mathrm{y} f_{c}^{\prime}$ en $\mathrm{kg} / \mathrm{cm}^{2}$

Las Normas Técnicas Complementarias del Distrito Federal, en su edición del 2004 (NTC-2004), establecen expresiones para el cálculo del módulo de elasticidad de los concretos en México, dividiéndolos en concretos clase I y clase II. Estas expresiones no han cambiado en las Normas Técnicas Complementarias para Diseño y Construcción de Estructuras de Concreto para la Ciudad de México (NTC, 2017). Para los concretos estructurales o de clase I se tiene:

$E_{c}=4400 \sqrt{f_{c}^{\prime}}, \quad E_{c}$ y $f_{c}^{\prime}$ en MPa

$E_{c}=14000 \sqrt{f_{c}^{\prime}}, \quad E_{c} \mathrm{y} f_{c}^{\prime}$ en $\mathrm{kg} / \mathrm{cm}^{2}$

para concretos de baja resistencia $\left(f^{\prime}{ }_{c}<40 \mathrm{MPa}\right), \mathrm{y}$

$E_{c}=2700 \sqrt{f_{c}^{\prime}}, \quad E_{c}$ y $f_{c}^{\prime}$ en MPa

$E_{c}=8500 \sqrt{f_{c}^{\prime}}, \quad E_{c} \mathrm{y} f_{c}^{\prime}$ en $\mathrm{kg} / \mathrm{cm}^{2}$

para concretos de alta resistencia $\left(f^{\prime}{ }_{c}>40 \mathrm{MPa}\right)$.

Para los concretos de clase II de acuerdo con las NTC-2017 se tiene:

$E_{c}=2500 \sqrt{f_{c}^{\prime}}, \quad E_{c}$ y $f_{c}^{\prime}$ en $\mathrm{MPa}$

$E_{c}=8000 \sqrt{f_{c}^{\prime}}, \quad E_{c} \mathrm{y} f_{c}^{\prime}$ en $\mathrm{kg} / \mathrm{cm}^{2}$

\section{Deformación $\varepsilon_{c}^{\prime}$}

En México no se han efectuado estudios para proponer expresiones que definan $\varepsilon_{c}{ }_{c}$ ó la relación $E_{c} / E_{s e c}$. Estudios de la estimación de $\varepsilon_{c}{ }_{c}$ han sido efectuados por Chen et al. (2013), quienes propusieron una expresión para $\varepsilon_{c}^{\prime}$ con base en información de ensayes de cilindros de concreto existentes en la literatura y realizando pruebas de laboratorio para complementarlo. Esta expresión, ec. 10, para obtener la deformación de $\varepsilon_{c}{ }_{c}$, ha sido utilizada en los últimos años debido a su similitud con los resultados obtenidos de ensayes en laboratorio, con un error de $\pm 10 \%$.

$$
\begin{aligned}
& \varepsilon_{c}^{\prime}=1.74 \times 10^{-7} f_{c}^{\prime}+2.41 \times 10^{-3}, \quad f_{c}^{\prime} \text { en MPa } \\
& \left(\varepsilon_{c}^{\prime}=1.71 \times 10^{-8} f_{c}^{\prime}+2.41 \times 10^{-3}, \quad f_{c}^{\prime} \text { en } \mathrm{kg} / \mathrm{cm}^{2}\right)
\end{aligned}
$$

De Nicolo et al. (1994), estudió el comportamiento de los concretos enfocándose en el estudio de la deformación asociada con el máximo esfuerzo en el concreto $\varepsilon^{\prime}$. Una aproximación de su expresión para el cálculo de $\varepsilon^{\prime}{ }_{c}$ para concretos con $f^{\prime}{ }_{c}$ entre $20 \mathrm{MPa}$ y $80 \mathrm{MPa}$, es la que se muestra en la ec. 11: 


$$
\begin{aligned}
& \varepsilon_{c}^{\prime}=10^{-6}\left(\frac{50}{3} f^{\prime}{ }_{c}+\frac{5000}{3}\right), \quad f^{\prime}{ }_{c} \text { en MPa } \\
& \left(\varepsilon_{c}^{\prime}=10^{-8}\left(\frac{490.5}{3} f^{\prime}{ }_{c}+\frac{500000}{3}\right), \quad f^{\prime}{ }_{c} \text { en } \mathrm{kg} / \mathrm{cm}^{2}\right)
\end{aligned}
$$

La revisión de los estudios efectuados en la literatura indica la complejidad que existe para estimar parámetros relevantes de la curva $f_{c}-\varepsilon_{c}$ del concreto en compresión. En el caso de normativas para la Ciudad de México, los estudios que avalan las expresiones utilizadas en la actualidad para obtener el módulo de elasticidad, $E_{c}$, tienen más de 30 años de antigüedad, y se basan en propiedades de concretos producidos en la Ciudad de México. Estas expresiones no serían aplicables para las diversas regiones de nuestro país, ya que no consideran los tipos de agregados existentes en estas regiones. Por lo tanto, es relevante realizar una investigación con fines de encontrar expresiones para estimar parámetros relevantes de la curva $f_{c}-\varepsilon_{c}$ para concretos producidos en México.

\section{DESCRIPCIÓN DE LOS ENSAYES REALIZADOS EN ESTE ESTUDIO}

La parte experimental de esta investigación se llevó a cabo en el laboratorio de Estructuras del Instituto de Ingeniería de la UNAM (II-UNAM), donde se ensayaron en compresión cilindros de concreto con resistencias especificadas $f^{\prime}{ }_{c}$ iguales a: $25 \mathrm{MPa}, 30 \mathrm{MPa}, 40 \mathrm{MPa}, 50 \mathrm{MPa}, 60 \mathrm{MPa}$ y $70 \mathrm{MPa}$. Para cada resistencia se contó con seis cilindros de concreto, lo que da un total de 36 cilindros por región del país. El país se dividió en 8 regiones que se describen más adelante. Lo anterior daría un total de 36 cilindros $\times 8$ regiones $=288$ cilindros de concreto de todo el país. El estudio final se avaló con alrededor de 200 cilindros, ya que no todas las regiones fabricaban todas las resistencias requeridas para este estudio. Después de los ensayes de cilindros de concreto se obtuvieron las curvas $f_{c}-\varepsilon_{c}$ para estos cilindros. Con base en los resultados obtenidos se proponen expresiones para la estimación tanto para $E_{c}$, como para $\varepsilon_{c}^{\prime} \mathrm{y}$ $E_{c} / E_{\text {sec }}$.

Debido a la gran extensión territorial de México, estudios anteriores, como los realizados por Mendoza (1985), han comprobado que los concretos tienen diferencias de acuerdo con la región donde han sido fabricados, por esto fue necesario realizar una zonificación del país con fines de muestreo para cubrir las variantes existentes en los concretos del país de acuerdo con el tipo de agregado y modo de fabricación (norma NMX-C-160, 2004). Esta zonificación se muestra en la fig. 3.

Las muestras con las que se realizó este estudio cumplieron la norma NMX-C-159 (NMX-C-159, 2004). Se utilizaron cilindros de $15 \mathrm{~cm} \times 30 \mathrm{~cm}$ para todas las resistencias, provenientes de las ciudades de: Tijuana, Chihuahua, Culiacán, Monterrey, Guadalajara, Ciudad de México, Acapulco y Veracruz; sin embargo, en esta investigación los resultados de los especímenes de Veracruz, que fueron dos, no se tomaron en cuenta por considerarse que no eran confiables. Con esta zonificación se consideró que se cubría una buena parte de las diferentes características de los concretos en México. Cuatro de las empresas fabricadoras de concreto más importantes del país, miembros de la AMIC, apoyaron la investigación, para lo cual donaron cilindros con las resistencias más comunes de plantas de concreto premezclado de las regiones, estos cilindros fueron enviados al laboratorio del Instituto de Ingeniería para realizar su ensaye correspondiente a 28 días. Para proporcionar las mezclas de concreto con las que se fabricaron los cilindros, así como su curado, estas empresas emplearon las normas NMX-C-159 (NMX-C-159, 2004) y NMX-C160 (NMX-C-160, 2004). Esto sugiere que la variabilidad de resultados de los ensayes efectuados con los cilindros fabricados por estas empresas sería comparable para todas ellas. 


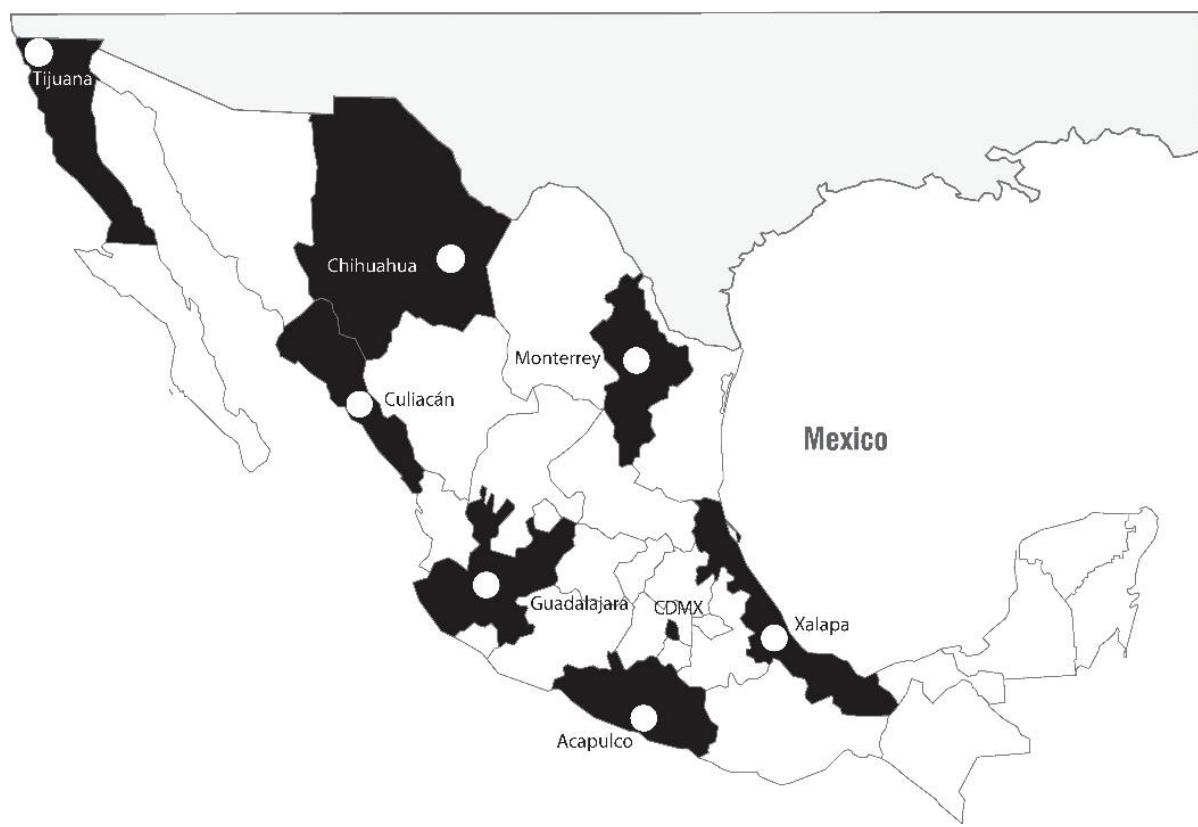

Figura 3. Zonificación para muestreos

Para las ciudades de Culiacán y Guadalajara se fabricaron concretos con agregado de "canto rodado" o bien grava de río, mientras que para las ciudades de Acapulco, Tijuana, Chihuahua y Monterrey el agregado grueso se obtuvo de la trituración de basaltos. Para la Ciudad de México el agregado fue de piedra caliza y para Xalapa fue de andesita. Los pesos volumétricos (P.V.) de los agregados variaron de 23.54 $\mathrm{kN} / \mathrm{m}^{3}$ a $26.38 \mathrm{kN} / \mathrm{m}^{3}$, para la grava, y de $22.36 \mathrm{kN} / \mathrm{m}^{3}$ a $26.48 \mathrm{kN} / \mathrm{m}^{3}$ para la arena, siendo los agregados de Veracruz los de valores más bajos y de Chihuahua los más altos.

Las muestras se obtuvieron de acuerdo con las resistencias que fabricaban a la fecha de este estudio (2015) las distintas plantas de concreto que colaboraron en este proyecto, por ello, las regiones estudiadas no cuentan con el mismo número de ensayes o las mismas resistencias de concretos. La Ciudad de México fue la única región con todas las resistencias deseadas en el estudio, debido a su mayor adelanto en la industria de la construcción respecto a otras regiones del país. En Rodelo et al. (2019) se puede encontrar una descripción detallada de las características de los cilindros de concreto ensayados, así como la instrumentación de éstos para obtener las curvas $f_{c}-\varepsilon_{c}$ para estos cilindros. El módulo de elasticidad $E_{c}$ se obtuvo siguiendo las especificaciones de la norma NMX-C-128 (NMX-C-128, 1997), la cual se basa en la norma ASTM-C-469 (ASTM-C-469, 2002). Una muestra consistía en tres cilindros fabricados en una región con la resistencia especificada del concreto en compresión. En esta muestra se obtuvo el promedio de resultados de estos tres cilindros para obtener parámetros que definen la curva $f_{c}-\varepsilon_{c}$ para esta muestra.

\section{EVALUACIÓN DE RESULTADOS PARA EL MÓDULO DE ELASTICIDAD $E_{c}$ OBTENIDOS DE LOS ENSAYES EN COMPRESIÓN DE CILINDROS DE CONCRETO PRODUCIDOS EN DIVERSAS REGIONES DE MÉXICO}

La fig. 4 muestra resultados encontrados para $E_{c}$ en los ensayes efectuados en este estudio con concretos producidos en diferentes regiones del país. La fig. 4 también muestra resultados para $E_{c}$ empleando diferentes predicciones. Para estimar el módulo elástico de concretos mexicanos con el ACI 31814 (ACI-318, 2014), ec. 5, se consideró para $w_{c}$ el valor promedio que fue igual a $2340 \mathrm{~kg} / \mathrm{m}^{3}$. Este procedimiento del ACI 318-14 lleva a sobrestimar los valores medidos de $E_{c}$ para altas resistencias, y 
subestimar los valores medidos para resistencias menores que aproximadamente $40 \mathrm{MPa}$. La fig. 4 muestra que los valores calculados para $E_{c}$ con el procedimiento de la NTC (2017) subestiman los valores experimentales obtenidos en este estudio. La fig. 4 también muestra que los resultados obtenidos con la ec. 4 del ACI-363 son comparables con los obtenidos con el procedimiento de la NTC (2017).

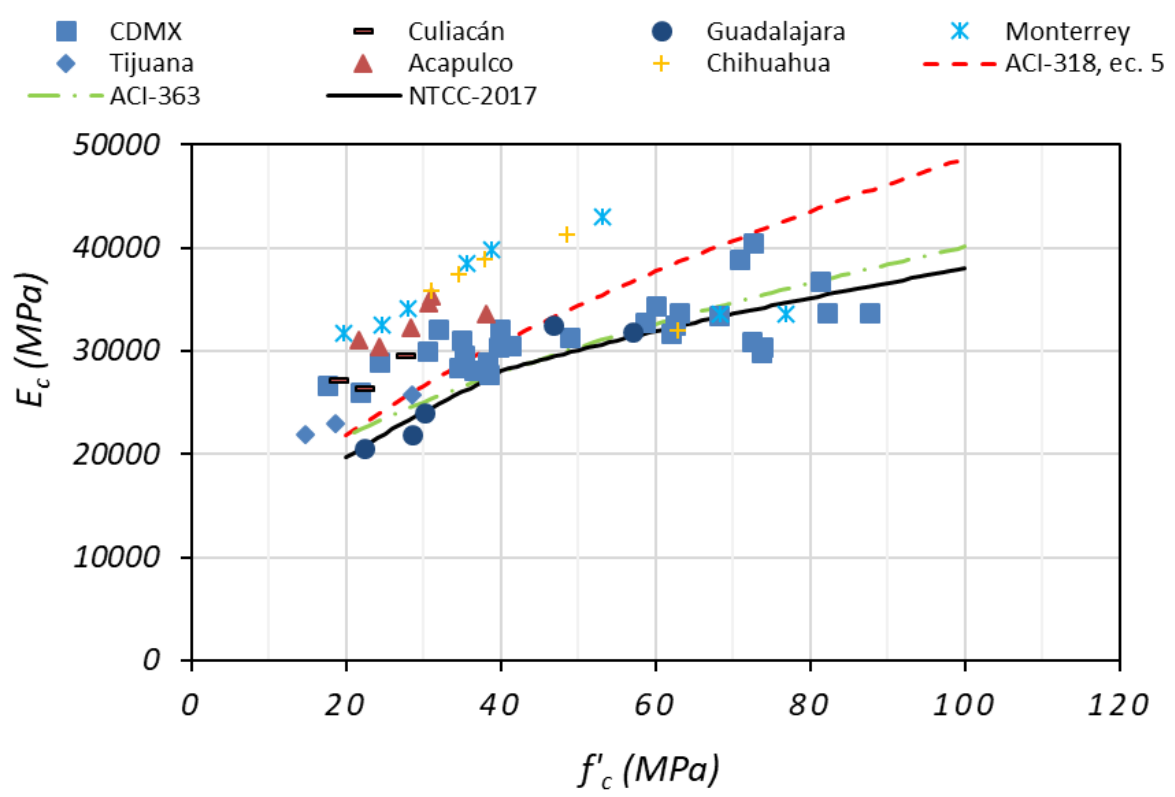

Figura 4. Comparaciones de valores de $E_{c}$ medidos y calculados con diversos procedimientos

En lo que sigue se muestran resultados para la relación $E_{c_{-} m} / E_{c_{-} \text {calc }}$ obtenidos con los resultados experimentales de este estudio donde $E_{c_{-} m}$ es el valor experimental de $E_{c}$ y $E_{c_{-} c a l c}$ es el valor obtenido de $E_{c}$ con estimaciones de diversos procedimientos.

\section{Procedimiento del ACI 318-14 con los valores de $w_{c}$ de cada espécimen}

La fig. 5 muestra resultados para la relación $E_{c_{-} m} / E_{c_{-} \text {calc }}$, donde $E_{c_{-} \text {calc }}$ se obtuvo empleando el ACI318-14 (2014), ec. 5, y el valor de $w_{c}$ de cada espécimen. Se puede apreciar que para concretos mayores de $40 \mathrm{MPa}$, los valores de Ec calculados sobrestiman de manera considerable los valores medidos. La media de $E_{c_{-} m} / E_{c_{-} \text {calc }}$ fue igual a 1.02 y con un valor del coeficiente de variación, CV, igual a 0.19 . Los valores mínimo y máximo para esta relación fueron iguales a 0.68 y 1.41, respectivamente.

\section{Procedimiento del ACI 318-14 para concretos de peso normal (ec. 6)}

La fig. 6 muestra valores de la relación $E_{c_{-} m} / E_{c_{-} \text {calc }}$, donde $E_{c_{-} \text {calc }}$ es el valor calculado de $E_{c}$ con el ACI-318 (2014) para peso normal, ec. 6. Los resultados siguen una tendencia similar al caso anterior empleando la ec. 5. La media de esta relación fue igual a 1.05, con un valor del CV igual a 0.20. Los valores mínimos y máximo para la relación $E_{c_{-} m} / E_{c_{\_} \text {calc }}$ son iguales a 0.73 y 1.51 , respectivamente. 


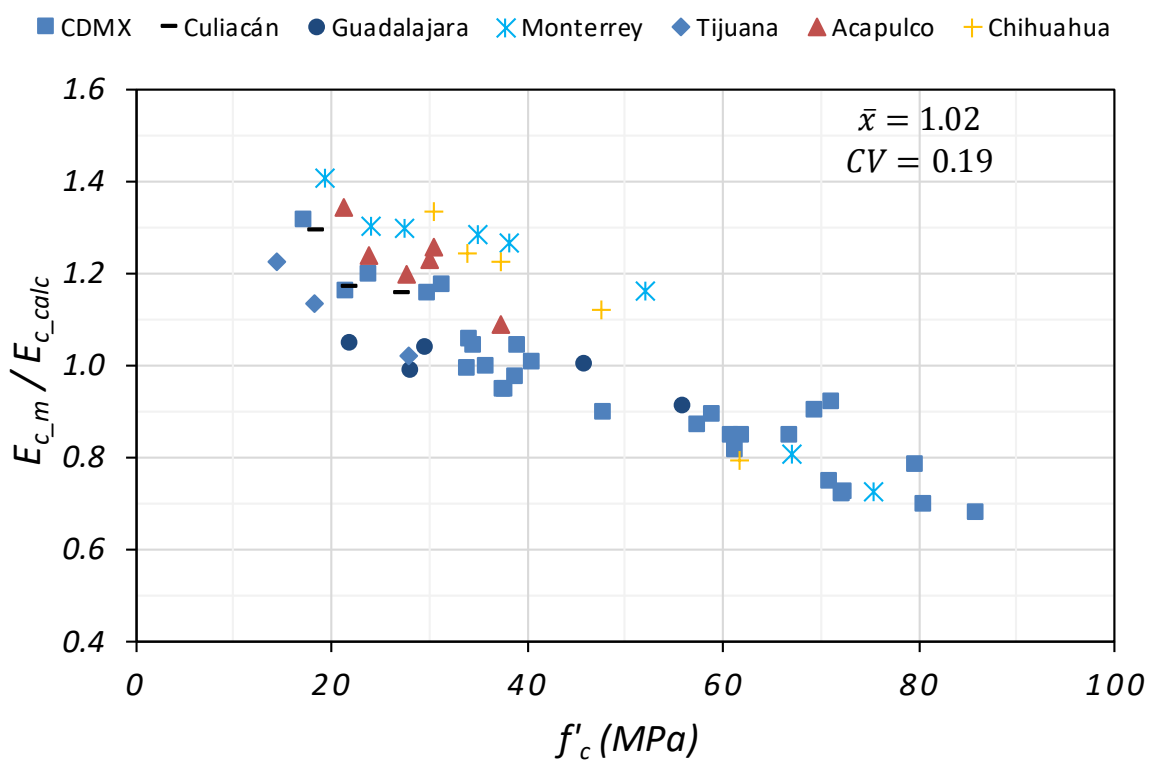

Figura 5. Relación $E_{c_{-} m} / E_{c_{-} \text {calc }}$ considerando el ACI 318-14, ec. 5

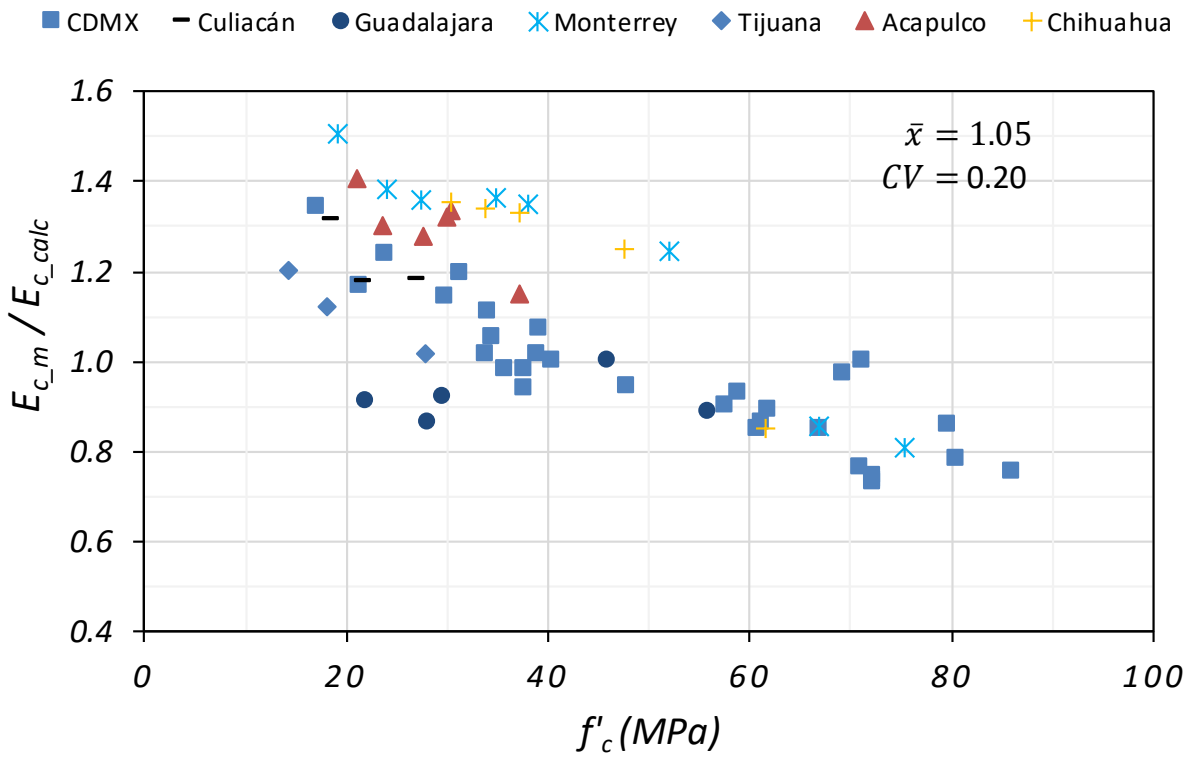

Figura 6. Relación $E_{c_{-} m} / E_{c_{\_} \text {calc }}$ considerando el ACI 318-14, ec. 6

\section{Procedimiento del ACI 363}

La fig. 7 muestra valores de la relación $E_{c_{-} m} / E_{c_{-} \text {calc }}$, donde $E_{c_{-} \text {calc }}$ es el valor calculado de Ec con el ACI 363, ec. 4. Estos resultados muestran que el procedimiento del ACI 363 lleva a subestimar los módulos elásticos en un intervalo amplio de $f^{\prime}$. La media de esta relación es igual a 1.11 y con un valor del CV igual a 0.16 . Los valores mínimos y máximo para esta relación son iguales a 0.83 y 1.45 , respectivamente. 


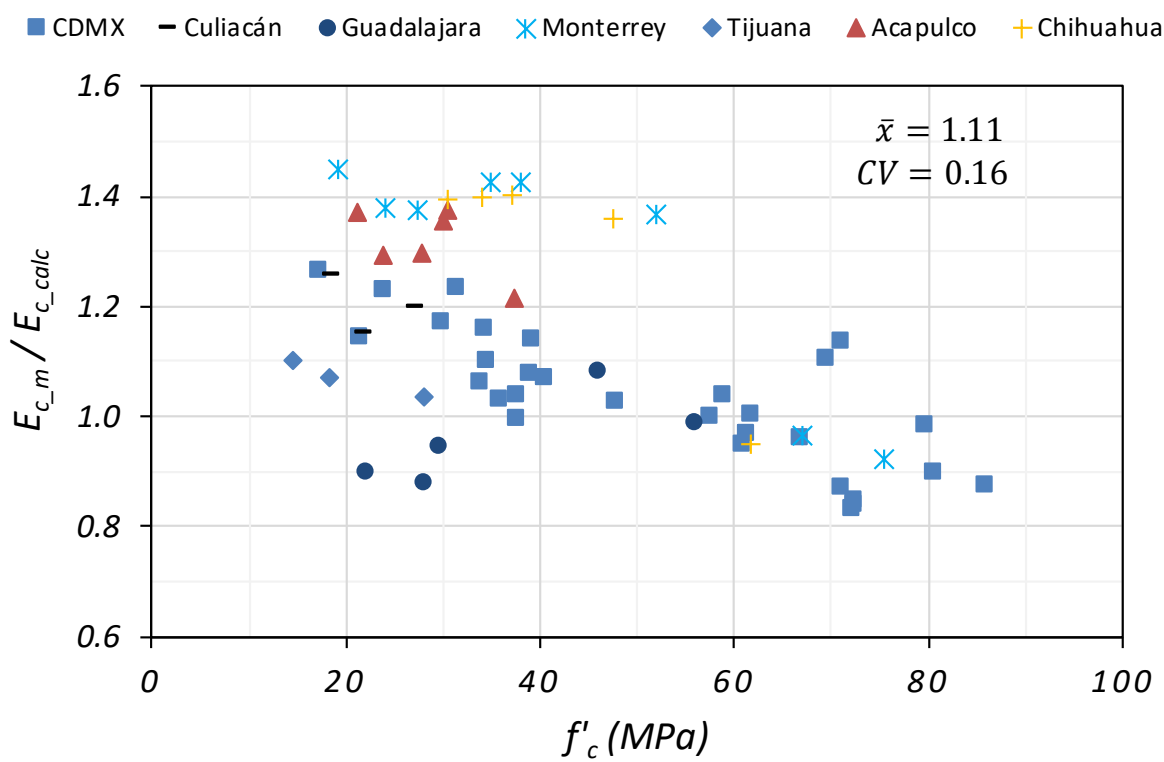

Figura 7. Relación $E_{c_{-} m} / E_{c_{\_} c a l c}$ considerando el ACI 363

\section{Procedimiento de la NTC 2017}

La fig. 8 muestra resultados para la relación $E_{c_{-} m} / E_{c_{-} \text {calc }}$, donde $E_{c_{-} \text {calc }}$ es el valor calculado de $E_{c}$ con las ecs. 7 y 8 de las Normas Técnicas Complementarias (NTC, 2017). En los resultados de la fig. 8 se consideraron solo concretos con valores de resistencias especificadas en compresión iguales o mayores que $25 \mathrm{MPa}$, estos concretos son los que se emplean en la fabricación de concretos clase 1 en la Ciudad de México (NTC, 2017). Estos resultados muestran que utilizar la NTC (2017), lleva a una variabilidad considerable y a la tendencia de sobreestimar para concretos de alta resistencia los valores medidos de $E_{c}$. La media de esta relación es igual a 1.11 y con un valor del CV igual a 0.17 . Los valores mínimos y máximo para esta relación son iguales a 0.86 y 1.45 , respectivamente.

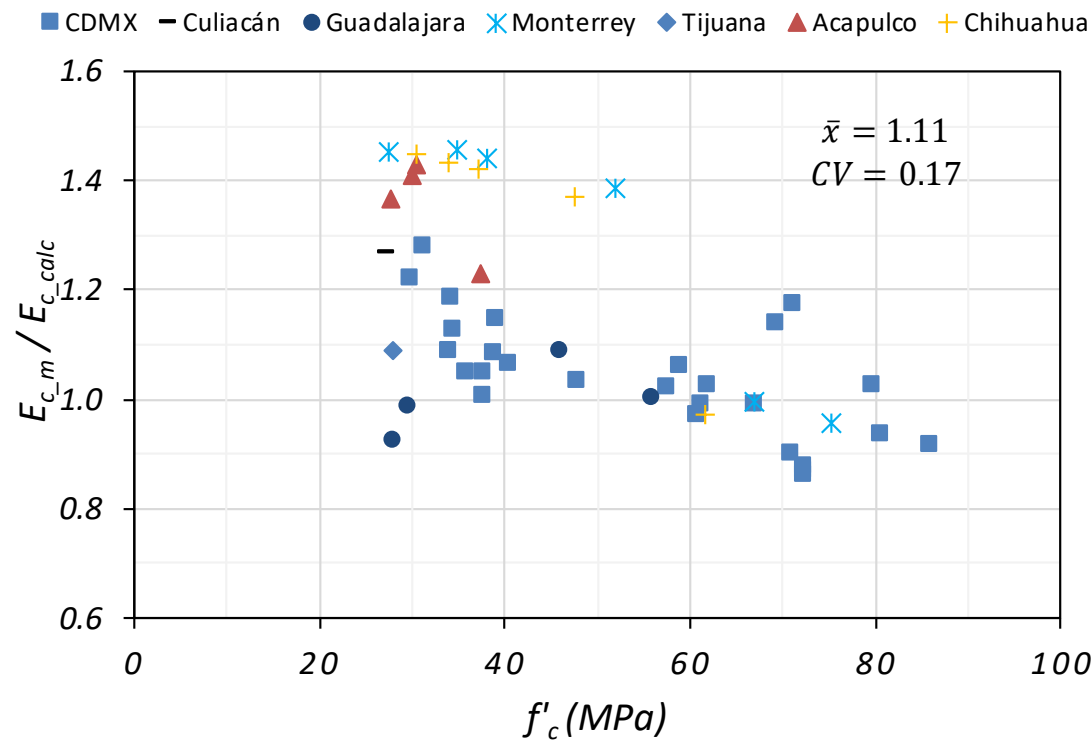

Figura 8. Relación $E_{c_{-} m} / E_{c_{-} \text {calc }}$ considerando la NTC 2017 
Los resultados muestran que el empleo de las NTC (2017) para estimar valores de $E_{c}$ de concretos del resto de las regiones, lleva a resultados con variación importante respecto a los resultados experimentales, la cual es más grande que la que se tendría con otros procedimientos. Estos resultados sugieren que para cualquiera de las regiones de México consideradas en este estudio, fuera de la Ciudad de México, de los procedimientos existentes para el cómputo de $E_{c}$ la mejor predicción se obtiene con el ACI 318 (2014).

\section{EXPRESIONES QUE SE PROPONEN EN ESTE ESTUDIO PARA LA PREDICCIÓN DE $E_{c}$}

La fig. 9(a) muestra resultados de $E_{c}$ medidos para la base de datos de las regiones estudiadas, excluyendo los casos de Chihuahua y Monterrey. El mejor ajuste estadístico a estos resultados para la relación $E_{c} / w_{c}^{1.5}$ lleva a la ec. 12 . En la ec. $12, E_{c}$ y $f_{c}{ }_{c}$ tienen unidades en MPa y $w_{c}$ en $\mathrm{kg} / \mathrm{m}^{3}$. En paréntesis se muestra la expresión a usar en unidades de sistema métrico. La fig. 9(b) muestra resultados para la población total de cilindros de concreto incluyendo los casos de Chihuahua y Monterrey. Como se aprecia en ella, la ec. 12 lleva a subestimar los valores de $E_{c} / w_{c}^{1.5}$ para la mayor parte de los casos de Chihuahua y Monterrey. Esto sugiere que el tipo agregado de estas últimas regiones sería factor importante para esta subestimación de los valores calculados respecto a los experimentales.

$$
\begin{aligned}
& E_{c}=0.14 w_{c}^{1.5}\left(f^{\prime}{ }_{c}\right)^{0.18}, \quad w_{c} \text { en } \mathrm{kg} / \mathrm{m}^{3} y{f^{\prime}}_{c} \text { en } \mathrm{MPa} \\
& \left(E_{c}=0.94 w_{c}^{1.5}\left(f^{\prime}{ }_{c}\right)^{0.18}, \quad w_{c} \text { en } \mathrm{kg} / \mathrm{m}^{3} y{f^{\prime}}_{c} \text { en } \mathrm{kg} / \mathrm{cm}^{2}\right)
\end{aligned}
$$

La fig. 10 permite comparar valores de la relación $E_{c_{-} m} / E_{c_{-} \text {calc }}$ utilizando para $E_{c_{-} \text {calc }}$ la ec. 12 con los valores de $w_{c}$ de cada espécimen. La fig. 10 se divide en dos figuras, la fig. 10(a) nuevamente excluye a los casos de C hihuahua y Monterrey, y la fig. 10(b) corresponde a todos los casos estudiados. Para el primer caso, la media entre los valores medidos y calculados fue 0.98 , y el valor de CV fue igual a 0.08 . Los valores mínimo y máximo para esta relación fueron 0.87 y 1.15 , respectivamente. Cuando se consideran todos los casos de la base de datos, la media entre los valores medidos y calculados fue 1.01, y el valor de CV fue igual a 0.11. Los valores mínimo y máximo para esta relación fueron 0.87 y 1.27, respectivamente. Estos resultados indican que la expresión propuesta, ec. 12, lleva a resultados con mejores parámetros estadísticos que los correspondientes al caso del empleo del ACI 318, (2014), ec. 5, para el cual la media y CV fueron iguales a 1.02 y 0.19 , respectivamente. 
Parámetros relevantes de la curva esfuerzo-deformación en compresión de concretos no confinados producidos en México

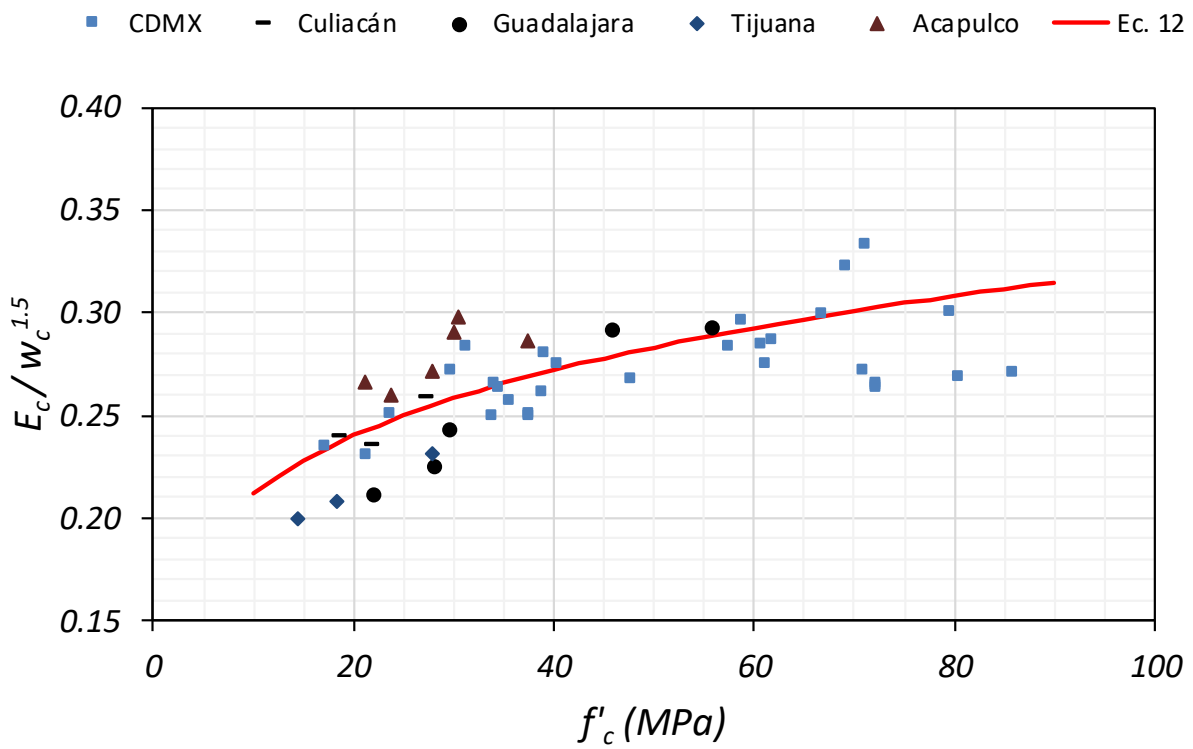

a) Excluyendo datos de Chihuahua y Monterrey

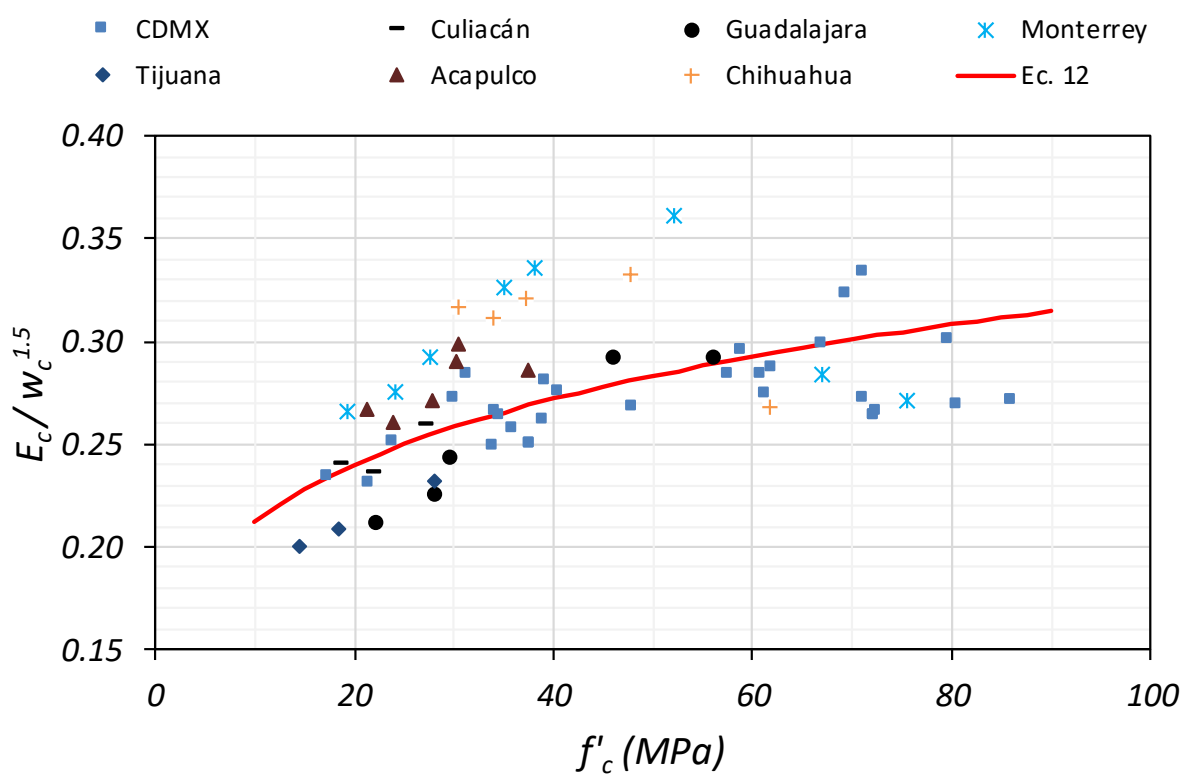

b) Incluyendo todos los datos

Figura 9. Valores medidos y calculados de $E c / w c^{1.5}$ empleando la ec. 12. 


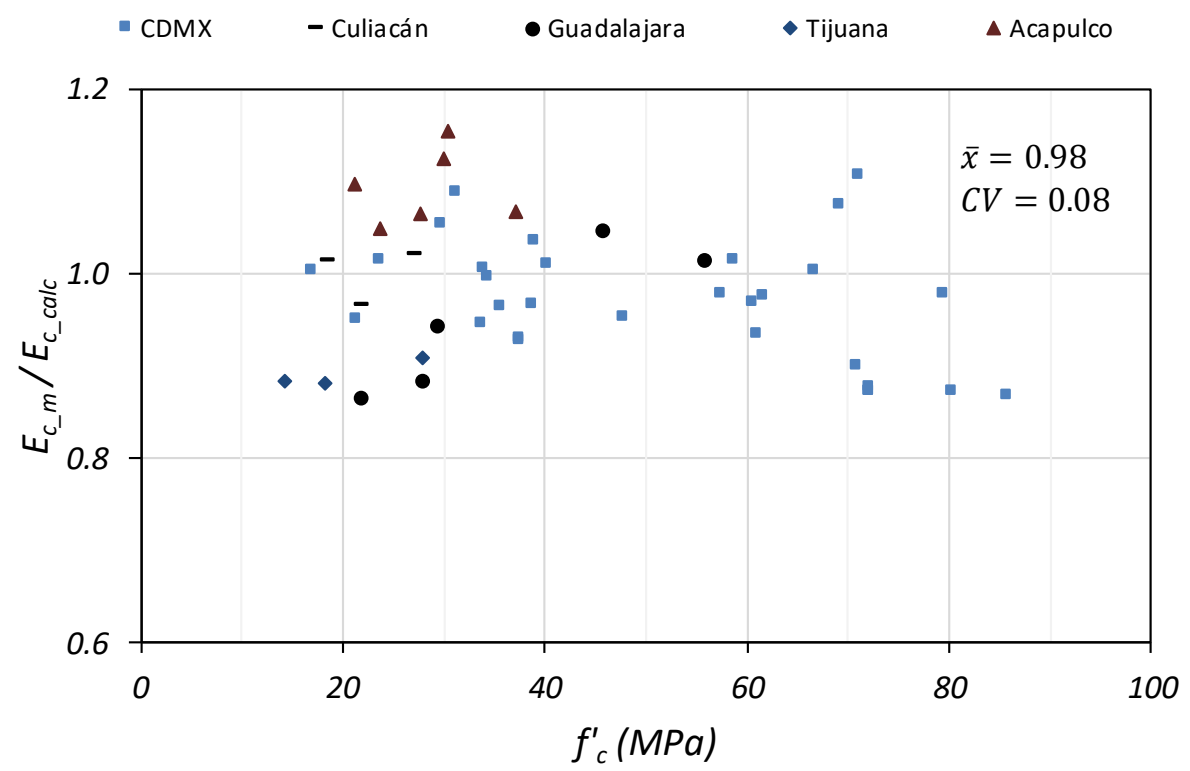

a) Excluyendo datos de Chihuahua y Monterrey

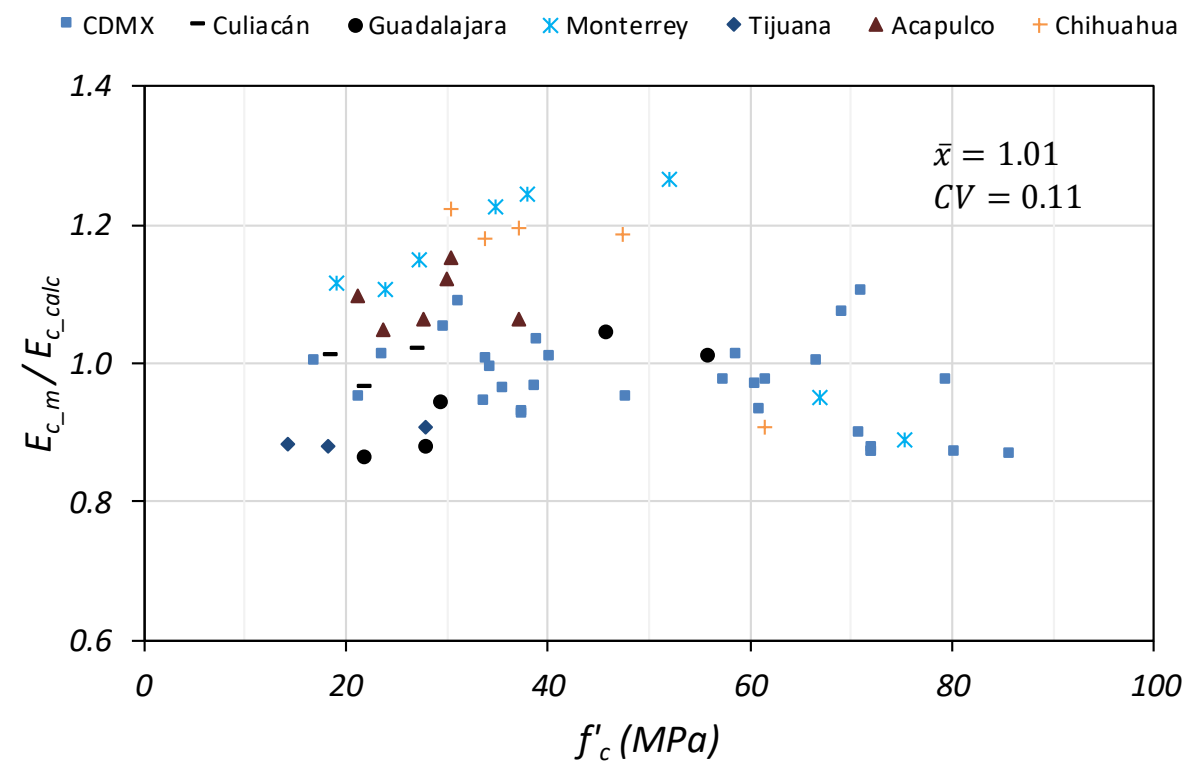

b) Incluyendo todos los datos

Figura 10. Relación $E_{c_{-} m} / E_{c_{-} c a l c}$ empleando $w_{c}$ medido, ec. 12.

La ec. 13 corresponde al caso del empleo de la ec. 12 reemplazando $w_{c}$ para concretos de peso normal (aproximadamente $2320 \mathrm{~kg} / \mathrm{m}^{3}$ ), lo que lleva a:

$$
\begin{aligned}
& \left(E_{c}=16000\left(f_{c}^{\prime}\right)^{0.18,} \quad f_{c}^{\prime} \text { en } \mathrm{MPa}\right) \\
& \left(E_{c}=105000\left(f_{c}^{\prime}\right)^{0.18,} \quad f_{c}^{\prime} \text { en } \mathrm{kg} / \mathrm{cm}^{2}\right)
\end{aligned}
$$


La fig. 11 muestra valores de la relación $E_{c_{-} m} / E_{c_{-} \text {calc }}$, empleando para $E_{c_{-} \text {calc }}$ la ec. 13 para concretos de peso normal. Esta figura se divide en dos figuras, la fig. 11(a) excluye a los casos de Chihuahua y Monterrey, y la fig. 11(b) corresponde a todos los casos estudiados. Para el primer caso, la media entre los valores medidos y calculados fue 0.98 , y el valor de CV fue igual a 0.10 . Los valores mínimo y máximo para esta relación fueron 0.74 y 1.20 , respectivamente. Cuando se consideran todos los casos de la base de datos, la media entre los valores medidos y calculados fue 1.02, y el valor de CV fue igual a 0.13 . Los valores mínimo y máximo para esta relación fueron 0.74 y 1.32 , respectivamente.

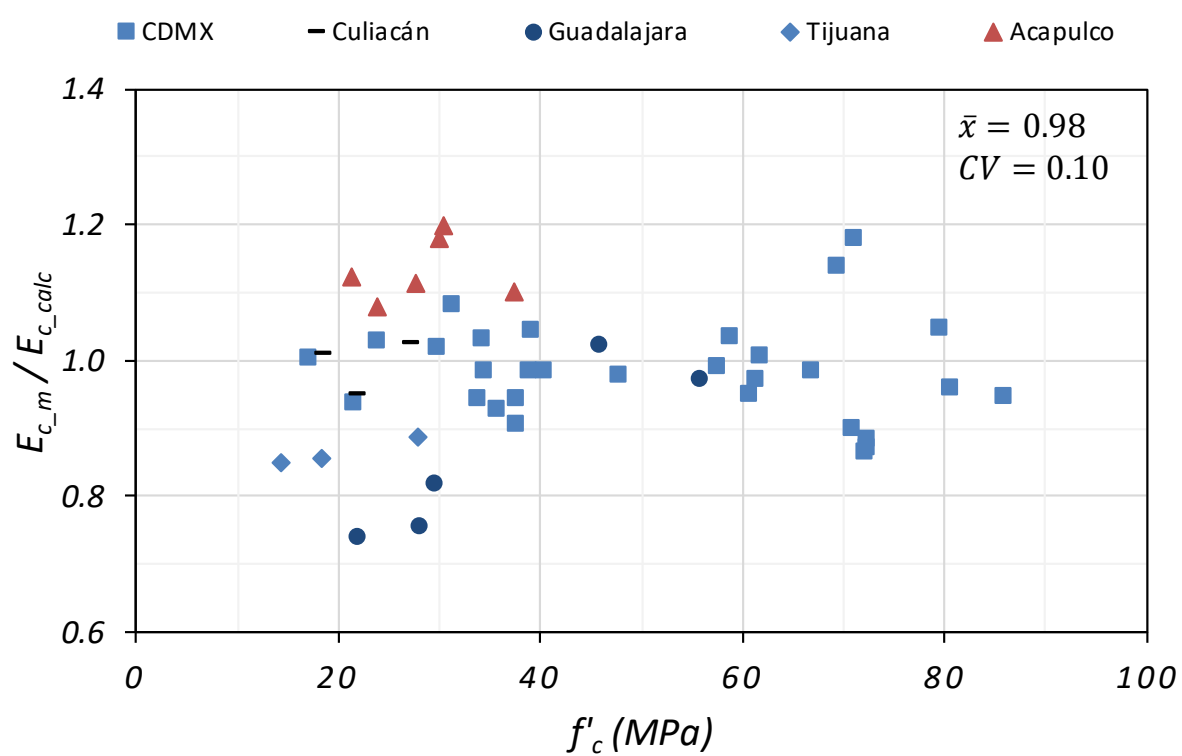

a) Excluyendo datos de Chihuahua y Monterrey

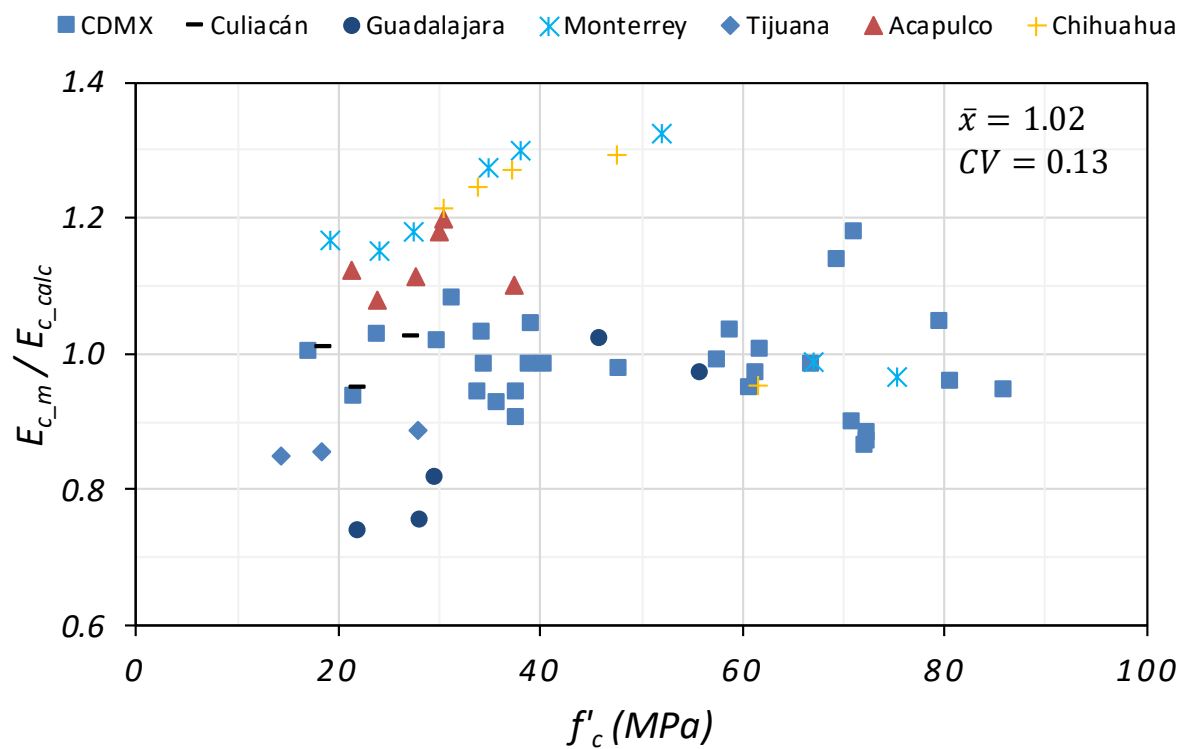

b) Incluyendo todos los datos

Figura 11. Relación $E_{c_{-} m} / E_{c_{-} \text {calc }}$ empleando para $w_{c}$ un valor promedio, ec. 13. 
Los resultados encontrados indican que el empleo de la ec. 13 para concretos de peso normal lleva a resultados con parámetros estadísticos que indican mejor predicción de $E_{c}$ comparado con los que se tendrían empleando las expresiones del ACI 318 (2014) comentados anteriormente.

Comparativa de valores de relación $\mathbf{E}_{\mathbf{c}_{-} m} / \mathbf{E}_{\mathrm{c}_{\_} \text {calc }}$ empleando diversos criterios para $\mathrm{E}_{\mathrm{c}_{\text {_calc }}}$ considerados en esta investigación

La fig. 12 muestra resultados de probabilidades acumuladas encontrados para la relación $E_{c_{\_} m} / E_{c_{-} \text {calc }}$ empleando diversos criterios para $E_{c_{-} \text {calc }}$. Estos resultados indican que las mejores predicciones para evaluar $E_{c}$ para la base de datos estudiada se obtienen empleando las ecs. 12 y 13 propuestas en este estudio. La fig. 12 muestra además que las expresiones de las NTC 2017 para Ec llevan a la mayor dispersión respecto a los valores medidos de $E_{c}$.

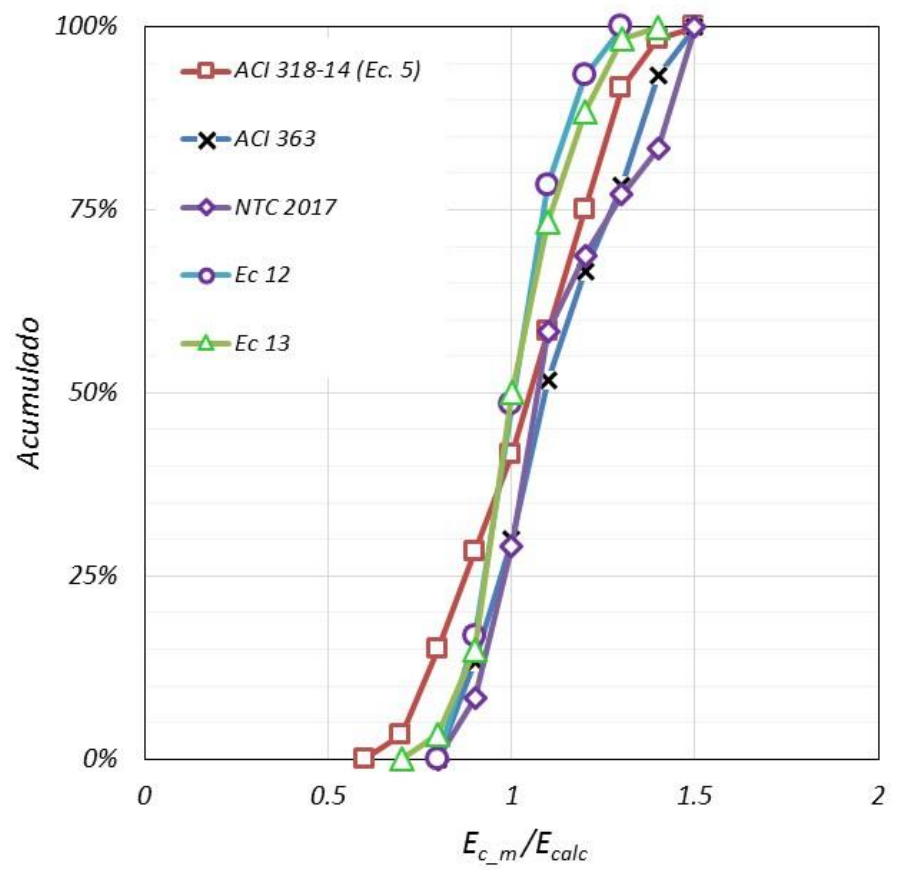

Figura 12. Probabilidades acumuladas encontrados para la relación $E_{c_{-} m} / E_{c_{-} \text {calc }}$ empleando diversos criterios para $E_{c_{-} \text {calc. }}$.

\section{EXPRESIONES QUE SE PROPONEN EN ESTE ESTUDIO PARA LA PREDICCIÓN DE LA RELACIÓN $E_{c} / E_{s e c}$}

A la relación $E_{c} / E_{s e c}$ en la literatura también se le denomina, $n$. La fig. 13 muestra resultados con marcadores obtenidos para esta relación en función de $f_{c}^{\prime}$, para la base de datos de concretos producidos en México empleada en esta investigación. Con línea continua la fig. 13 muestra resultados de aplicar la ec. 14 propuesta:

$$
\begin{aligned}
& n=1+\frac{25}{f_{c}^{\prime}}, \quad f_{c}^{\prime} \text { en } M P a \\
& \left(n=1+\frac{255}{f_{c}^{\prime}}, \quad f_{c}^{\prime} \text { en } \mathrm{kg} / \mathrm{cm}^{2}\right.
\end{aligned}
$$


Parámetros relevantes de la curva esfuerzo-deformación en compresión de concretos no confinados producidos en México

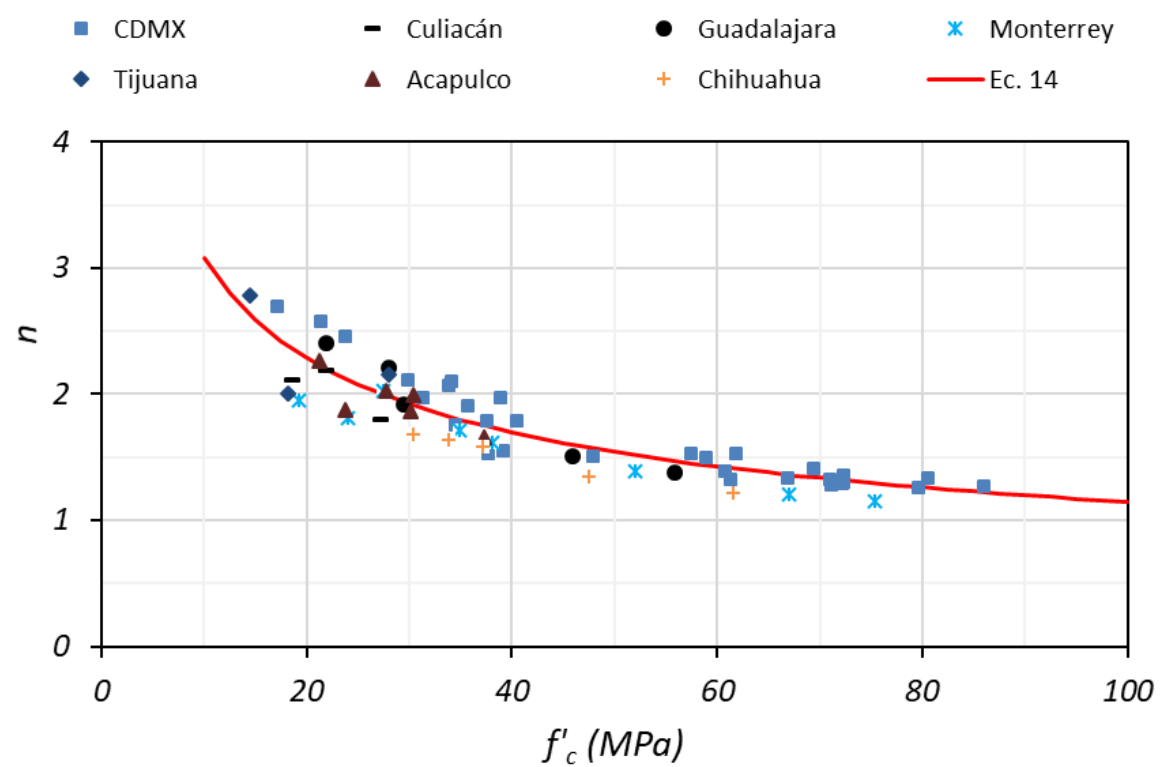

Figura 13. Parámetro $n$ vs $f^{\prime}{ }_{c}$

La fig. 14 muestra la relación entre el valor de $\mathrm{n}$ medido, $n_{m}$, versus el valor de $\mathrm{n}$ calculado, $n_{c}$, en este caso obtenido con la ec. 14. La media y CV para la relación $n_{m} / n_{c}$ fueron 1.01 y 0.096 , respectivamente. Los valores mínimo y máximo para esta relación fueron 0.85 y 1.21 , respectivamente.

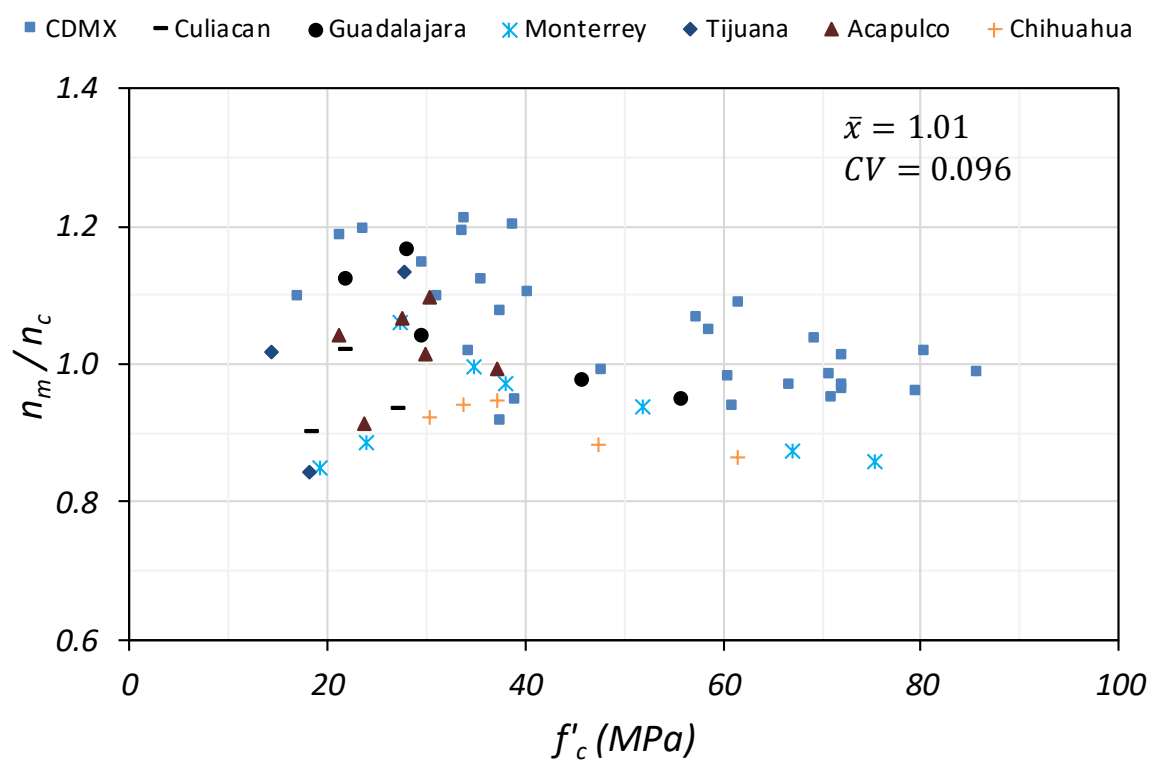

Figura 14. Relación entre $n_{m}$ y $n_{c}$ 


\section{PROCEDIMIENTO QUE SE PROPONE EN ESTE ESTUDIO PARA LA PREDICCIÓN DEFORMACIÓN $\varepsilon^{\prime}$}

De la definición de la deformación $\varepsilon_{c}{ }_{c}$ se tiene:

$\varepsilon_{c}^{\prime}=\frac{n f_{c}}{E_{c}}$

Reemplazando en la ec. 15 el valor de $n$ dado por la ec. 14 , se obtiene:

$\varepsilon_{c}^{\prime}=\frac{f_{c}^{,}+25}{E_{c}} \quad f_{c}$ en $M P a$

La fig. 15 muestra resultados de $\varepsilon^{\prime} c$ obtenidos de mediciones en el Laboratorio de Estructuras del Instituto de Ingeniería de la UNAM, indicados con marcadores. La fig. 15 muestra además resultados obtenidos con la ec. 11 (modificada de la propuesta por De Nicolo et al. 1994), indicados con línea continua. Con línea punteada se indican los resultados obtenidos con la ec. 16 empleando para el cómputo de $E_{c}$ la ec. 13 en unidades SI.

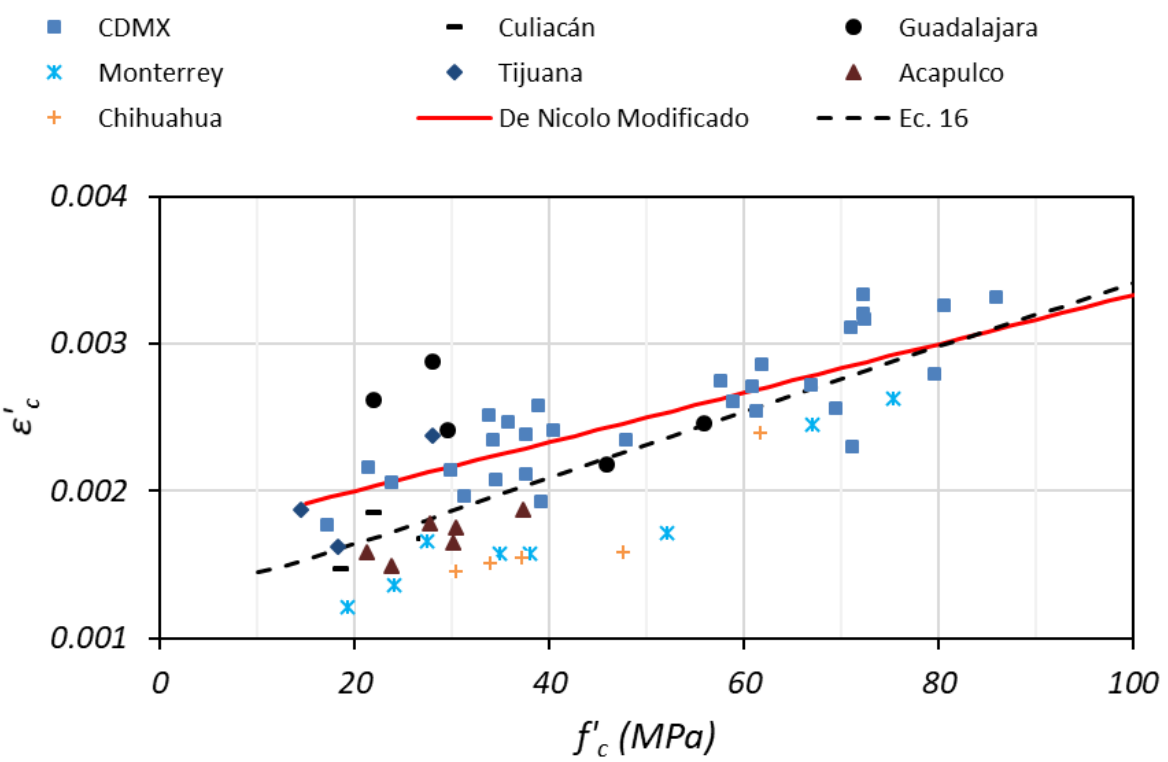

Figura 15. Valores medidos y calculados para la deformación $\varepsilon_{c}^{\prime}$ en función de $f_{c}^{\prime}$

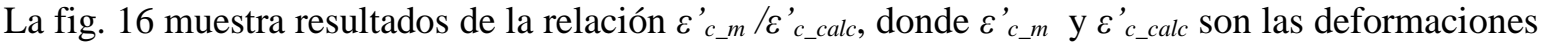
$\varepsilon_{c}^{\prime}$ medidas y calculadas con la ec. 16 , respectivamente, donde en esta ecuación se empleó el valor de $E_{c}$ dado por la ec. 13. Para esta relación, la media y CV fueron 1.01 y 0.185 , respectivamente. Los valores mínimo y máximo para esta relación son iguales a 0.70 y 1.58 , respectivamente. Se debe mencionar que la media y CV para la relación $\varepsilon_{{ }_{c \_} m} / \varepsilon^{\prime}{ }_{c_{-} \text {calc }}$ empleando la ec. 11 (modificada de De Nicolo et al., 1994) son iguales a 0.91 y 0.184, respectivamente, con valores mínimos y máximos iguales a 0.61 y 1.35 , respectivamente, lo que indica que la expresión propuesta para $\varepsilon_{c}^{\prime}$ da mejores resultados que la de De Nicolo et al. (1994). 


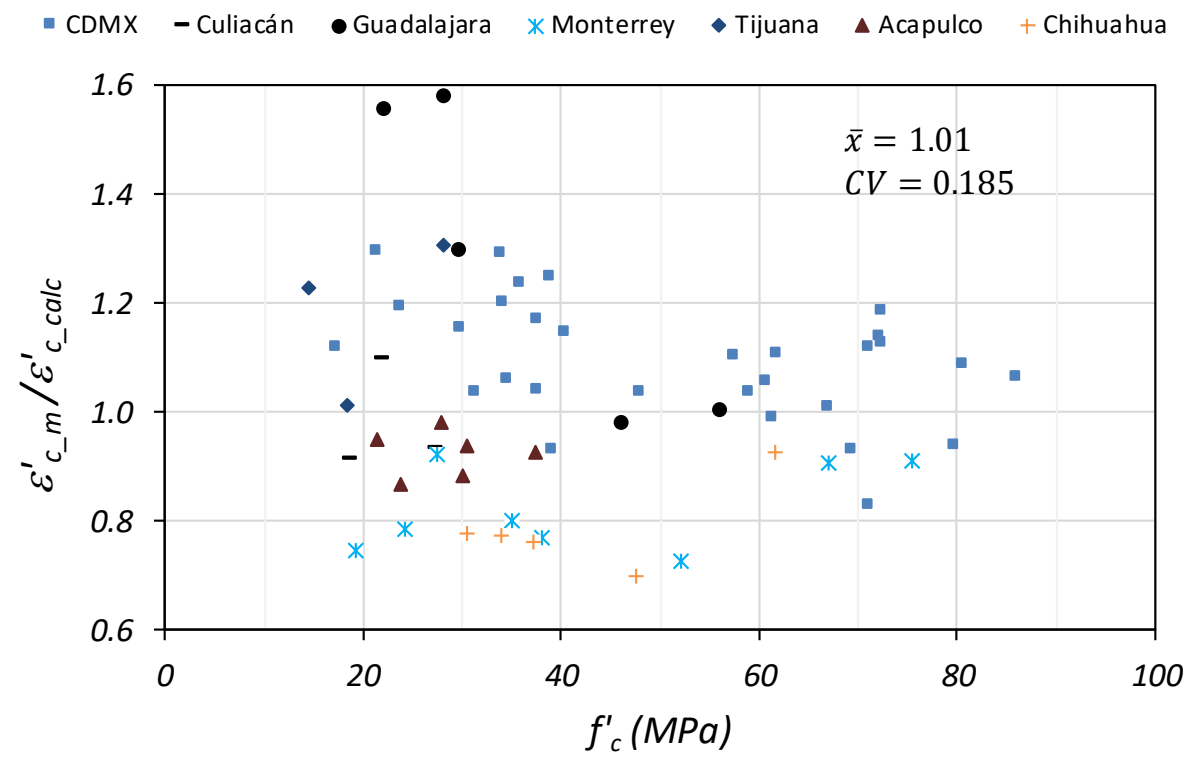

Figura 16. Relación $\varepsilon^{\prime}{ }_{c_{-} m} / \varepsilon^{\prime}{ }_{c_{-}}$calc considerando la expresión propuesta en este estudio.

\section{CONCLUSIONES}

En esta investigación se estudiaron parámetros relevantes que definen la curva-esfuerzo-deformación en compresión del concreto sin confinar, $f_{c}-\varepsilon_{c}$, para lo cual se emplearon resultados de ensayes en compresión de 200 cilindros de concreto con diversas resistencias especificadas de $f^{\prime}$ c. Estos cilindros de concretos fueron producidos por plantas de concreto en ciudades de diferentes regiones del país, para lo cual se siguieron las normas ASTM y NMX correspondientes, como se describe en este estudio. Estas ciudades fueron Tijuana, Chihuahua, Culiacán, Monterrey, Guadalajara, Ciudad de México y Acapulco. Los valores de $f_{c}^{\prime}$ estuvieron en el intervalo $20 \mathrm{MPa}$ a $100 \mathrm{MPa}$. En este estudio se obtuvieron las siguientes conclusiones.

1. La relación entre los valores medidos de $E_{c}$ para la base de datos de concretos producidos en México y los valores calculados con la ec. 13 propuesta en este estudio para concretos de peso normal tuvo una media de 1.02 y un CV igual a 0.13. La predicción simplificada dada por la ec. 13 propuesta fue mejor que la obtenida con la expresión del ACI 318-14 (ACI 318, 2014) para concretos de peso normal. La relación entre los valores medidos de $E_{c}$ para la base de datos y los valores calculados con esta expresión del ACI 318-14 tuvo una media de 1.05 y un CV igual a 0.20. Las expresiones de la NTC 2017 para $E_{c}$ para la base de datos no llevaron a predicciones mejores que los obtenidos con la ec. 13 o con el ACI 318-14. La relación entre los valores medidos de $E_{c}$ para la base de datos y los valores calculados con las expresiones de la NTC 2017 tuvo una media de 1.11 y un CV igual a 0.17.

2. La expresión propuesta para el cómputo de la relación $E_{d} / E_{s e c}$, ec. 14, lleva a una buena predicción de esta relación para base de datos de concretos producidos en México, tanto para concretos de baja resistencia como para las resistencias más altas encontradas en la literatura. La media y CV de los valores de $E_{d} / E_{s e c}$ empleando la ec. 14 fueron iguales a 1.01 y 0.096 , respectivamente. 
3. Se propone la ec. 16 para estimar $\varepsilon_{c}^{\prime}$. La media y CV de los valores de $\varepsilon_{c}^{\prime}$ empleando la ec. 16 son iguales a 1.01 y 0.185 , respectivamente. Esta expresión lleva a mejores resultados que con la propuesta de De Nicolo et al. (1994).

\section{AGRADECIMIENTOS}

Esta investigación fue patrocinada por el Instituto de Ingeniería, en su convocatoria 2014 para el financiamiento de Proyectos Internos. Se agradece la generosa colaboración de la Asociación Mexicana de la Industria del Cemento Premezclado A.C., quienes construyeron y donaron los cilindros de concreto entregados en el Instituto de Ingeniería para esta investigación. Sin esa colaboración, este estudio no hubiera sido posible. Se agradece la contribución de la Universidad de California en San Diego, quien permitió la estancia en esa Universidad del M. en I. Ramón Rodelo, Se agradece a David Solís por su ayuda en la interpretación de la base de datos considerada en esta investigación.

\section{REFERENCIAS}

ACI-318 (2014). Building Code Requirement for Reinforced Concrete. American Concrete Institute.

ACI-363 (1992). Report on High-Strength Concrete. American Concrete Institute Committee 363.

ASTM-C-469 (2002). Standard Test Method for Static Modulus of Elasticity y Poisson's Ratio of Concrete in Compression. American Society for Testing Materials.

Chen, Y, D J Visintin y U J Alengaram (2013). "Size-dependent stress-strain model for unconfined concrete". Structural Engineering, ASCE, 14(5), 11. https://doi.org/10.1061/(ASCE)ST.1943541X.0000869

De Nicolo, B, L Pani, y E Pozzo (1994). "Strain of concrete at peak compressive stress for a wide range of compressive strengths". Mater. Struct 27, (pp. 206-210). https://doi.org/10.1007/BF02473034

Mendoza, C J (1985). "Propiedades mecánicas de los concretos fabricados en el Distrito Federal”. Instituto de Ingeniería UNAM.

NMX-C-128 (1997). “Determinación del módulo de elasticidad estático y relación de Poisson”. Organismo Nacional de Normalización y Certificación de la Construcción y Edificación, S.C.

NMX-C-159 (2004). "Elaboración y curado de especímenes en el laboratorio". Organismo Nacional de Normalización y Certificación de la Construcción y Edificación, S.C.

NMX-C-160 (2004). "Elaboración y curado de especímenes de concreto". Organismo Nacional de Normalización y Certificación de la Construcción y Edificación, S.C.

NTC (2004). "Normas técnicas complementarias para diseño y construcción de estructuras de concreto", Gaceta Oficial de Distrito Federal.

NTC (2017). "Normas técnicas complementarias para diseño y construcción de estructuras de concreto", Gaceta Oficial de la Ciudad de México.

NTCS (2017). "Normas técnicas complementarias para diseño por sismo", Gaceta Oficial de la Ciudad de México.

Rodelo, R, M Rodriguez, y J Restrepo (2019), "Estudio de las características de la curva esfuerzodeformación de concretos típicos en México", Series del Instituto de Ingeniería, SID 706, https://aplicaciones.iingen.unam.mx/ConsultasSPII/DetallePublicacion.aspx?id=5182 